1999

\title{
Ion Heating In The Helix Helicon Plasma Source
}

\author{
J. L. Kline \\ E. E. Scime \\ P. A. Keiter \\ M. M. Balkey \\ R. F. Boivin
}

Follow this and additional works at: https://researchrepository.wvu.edu/faculty_publications

\section{Digital Commons Citation}

Kline, J. L.; Scime, E. E.; Keiter, P. A.; Balkey, M. M.; and Boivin, R. F., "Ion Heating In The Helix Helicon Plasma Source" (1999). Faculty Scholarship. 571.

https://researchrepository.wvu.edu/faculty_publications/571 


\title{
Ion heating in the HELIX helicon plasma source
}

\author{
J. L. Kline, E. E. Scime, P. A. Keiter, M. M. Balkey, and R. F. Boivin \\ Department of Physics, West Virginia University, Morgantown, West Virginia 26506
}

(Received 16 February 1999; accepted 26 August 1999)

\begin{abstract}
Efficient ion heating in a steady-state helicon plasma source is observed with two external loop antennae just above the ion cyclotron frequency. The ion velocity space distribution is measured by laser induced fluorescence in an argon plasma. The measured bulk ion heating is highly anisotropic (the perpendicular temperature increase is ten times the parallel temperature increase) even though the plasma is moderately collisional. Measurements of the perturbed distribution function with laser induced fluorescence suggest that an electrostatic ion cyclotron wave is launched. (c) 1999 American Institute of Physics. [S1070-664X(99)02212-0]
\end{abstract}

\section{INTRODUCTION}

Although primarily considered to be a plasmaprocessing device, the helicon source has a number of features that make it attractive for basic plasma physics experiments, e.g., high density $\left(n \approx 10^{13} \mathrm{~cm}^{-3}\right)$, steady-state operation, electrodeless plasma production, and modest magnetic fields. Helicon source groups around the world are actively investigating the high ionization efficiency of the helicon source $;^{1-3}$ the structure of the helicon wave in the plasma, ${ }^{4-6}$ the utility of heliconlike sources for fusion experiments; ${ }^{7}$ and the application of helicon sources for space-relevant, laboratory plasma physics experiments. ${ }^{8,9}$

Because of their high plasma densities, helicon sources are capable of producing fully magnetized, high beta $(\beta$ $=8 \pi n k T / B^{2}$ ) plasmas when the source is coupled to a large, low magnetic field chamber. ${ }^{9}$ Electromagnetic instabilities driven by ion temperature anisotropy are believed to play a significant role in the redistribution of energy in the Earth's magnetosheath. ${ }^{10,11}$ To investigate these instabilities in a controlled environment, the West Virginia University Hot hELicon eXperiment (HELIX) was constructed to generate high beta, fully magnetized plasmas with a controllable level of ion temperature anisotropy in the Large Experiment on Instabilities and Anisotropies (LEIA) space simulation chamber.

Recent measurements indicate that argon HELIX plasmas have a substantial intrinsic ion temperature anisotropy $\left(T_{\perp} / T_{\|}>4\right)$ and that the anisotropy depends on the strength of the HELIX magnetic field. ${ }^{8}$ A similar correlation between perpendicular ion temperature and magnetic field strength has been observed in the $\mathrm{H}-1$ heliac device (parallel ion temperatures have not been measured in $\mathrm{H}-1){ }^{12}$ The dependence of the intrinsic ion temperature on rf power. rf frequency, and neutral pressure was also investigated in previous experiments. ${ }^{8}$ To implement control of the ion temperature anisotropy without changing the HELIX magnetic field, an auxiliary ion heating system was constructed for HELIX.

Many researchers have developed ion heating systems for fusion and small-scale laboratory experiments. Typically a wave-particle resonance is exploited to directly heat the ions, e.g., ion cyclotron, ${ }^{13-15}$ lower hybrid, ${ }^{16}$ Alfvén, ${ }^{16}$ upper hybrid, ${ }^{17}$ and ion Bernstein. ${ }^{18}$ Ion cyclotron resonant heating is the most common in tokamaks and linear devices. ${ }^{13,15,16}$ The greatest technical challenges to ion cyclotron resonant heating (ICRH) in a helicon source arise from the large ratio of the ion-ion collision frequency for typical helicon ion temperatures $\left(T_{i} \approx 0.3 \mathrm{eV}\right)$ to the ion gyrofrequency, $\nu_{i i} / \Omega_{i}$ $\sim 120$, and the modest magnetic field strengths $(B$ $\sim 1000 \mathrm{G}$ ) of helicon sources. The relatively high collision frequencies prevent the ions from completing any significant fraction of a gyro-orbit and the modest magnetic field strengths require RF sources and antennas that operate at very low frequencies ( $\Omega_{i} / 2 \pi \approx 38 \mathrm{kHz}$ for argon ions). ICRH has been accomplished at similar magnetic field strengths, e.g., Good et al. ${ }^{15}$ successively used a Nagoya type III antenna for ICRH in a low density, $n=5 \times 10^{9} \mathrm{~cm}^{-3}$, barium Q-machine plasma. For those experiments, $\nu_{i i} / \Omega_{i} \sim 0.3$. For typical tokamak operating parameters of $T_{i}=20 \mathrm{keV}$, $n=0.8 \times 10^{14} \mathrm{~cm}^{-3}$, and $B_{0}=5 \mathrm{~T}, \nu_{i i} / \Omega_{i}$ is approximately $2.5 \times 10^{-7}$.

In the work described here, we report the observation of ion heating in a collisional helicon source with a pair of rectangular loop antennae. The frequency for the ion heating lies just above the ion cyclotron frequency. The ion heating experiments were performed for two different helicon source antennae (a $19 \mathrm{~cm}$ and a $35 \mathrm{~cm}$ long, half-wave, right-handed helical antenna) and for two different neutral pressures (1.5 mTorr and 3.0 mTorr). With the $19 \mathrm{~cm}$ helicon antenna, the ion heating is maximum between the fundamental and second cyclotron harmonic at both 1.5 mTorr and 3.0 mTorr. Six times more heating is observed at 1.5 mTorr than at 3.0 mTorr. For the $35 \mathrm{~cm}$ helicon antenna, the amount of ion heating decreases considerably and a peak in the ion heating is seen only at 3.0 mTorr. Measurements of the perturbed distribution function ${ }^{19-21}$ suggest the presence of an electrostatic ion cyclotron wave. ${ }^{22}$ The distribution function measurements were also used to determine the amplitude of the electrostatic potential. At a single location on axis, the amplitude of the electrostatic potential is anticorrelated with the ion heating. 


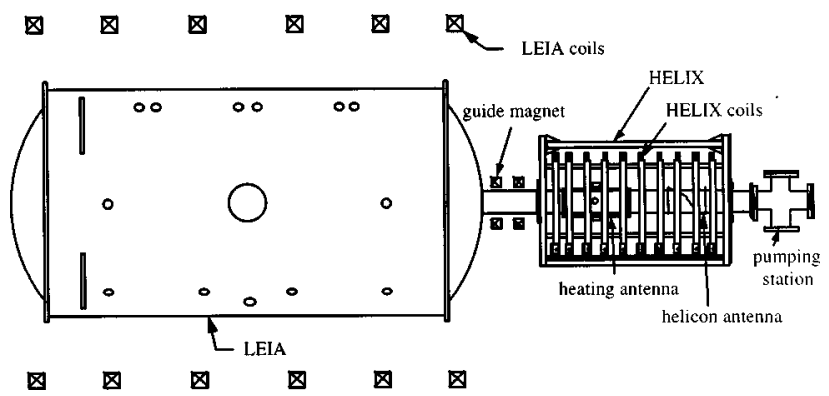

FIG. 1. The helicon source, HELIX, connected to LEIA. The ion heating antenna consists of two rectangular loops placed on either side of the HELIX Pyrex tube.

\section{EXPERIMENTAL APPARATUS}

The HELIX (Fig. 1) vacuum chamber is a Pyrex tube 15 $\mathrm{cm}$ in diameter and $157 \mathrm{~cm}$ long. Two pairs of opposing 2.5 $\mathrm{cm}$ ports in a cross formation located $52 \mathrm{~cm}$ from one end are used for laser induced fluorescence (LIF) measurements. Ten electromagnets produce a steady state axial magnetic field of $0-1300 \mathrm{G}\left(\Omega_{i} / 2 \pi=0-50 \mathrm{kHz}\right)$. The source gas is argon at neutral pressures of 1-10 mTorr. RF power of up to $2.5 \mathrm{~kW}$ over a frequency range of $6-20 \mathrm{MHz}$ is used to create the steady state plasma. The driving antennae used are a $19 \mathrm{~cm}$ and a $35 \mathrm{~cm}$ long, half-wave, right-handed helix. The righthandedness is relative to the magnetic field direction, and is designed to launch the $m=+1$ helicon wave towards LEIA. ${ }^{1,2}$ Characteristic electron temperature and densities in HELIX are $T_{e} \approx 5 \mathrm{eV}$ and $n \geqslant 1 \times 10^{13} \mathrm{~cm}^{3}$. For all the experiments reported here, the magnetic field in LEIA was fixed at $18 \mathrm{G}$. The ion temperatures, both parallel and perpendicular, are obtained from direct measurements of the ion velocity space distribution with LIF. ${ }^{8,23}$ Apart from Doppler and Zeeman broadening, all other line broadening mechanisms are ignorable for HELIX parameters. ${ }^{24}$ Whenever particle distribution functions are measured in a RF environment, ion sloshing (bulk motion of the entire ion distribution due to a rf electric field) is always a concern. ${ }^{25}$ At very low pressures, less than 1.5 mTorr, ion sloshing is occasionally observed in HELIX. All the measurements reported here are for fully thermalized ion distributions, i.e., changes in the measured ion temperatures correspond to changes in the thermal energy of the ions.

The $1000 \mathrm{~W}, 20-125 \mathrm{kHz}$ ion heating system was matched to the heating antenna with six, high-recirculatingcurrent, RF capacitors in an L network matching circuit. The real impedance for the entire circuit was less than $1 \Omega$ and the power delivered to the antenna was limited by the internal current limit of the RF generator. Since fixed capacitors were used, the RF frequency was held fixed at $36.5 \mathrm{kHz}$ and the HELIX magnetic field varied to search for the optimum magnetic field for ion heating. A number of different ion heating antenna geometries were investigated. ${ }^{24}$ These included Stix coils, ${ }^{26}$ single $m=0$ loops around the vacuum chamber, capacitively coupled plates, and transversely mounted loop antennas. Significant ion heating was observed for only the transversely mounted loop antennas. ${ }^{24}$ The transverse antennas consisted of two circular or rectangular loops
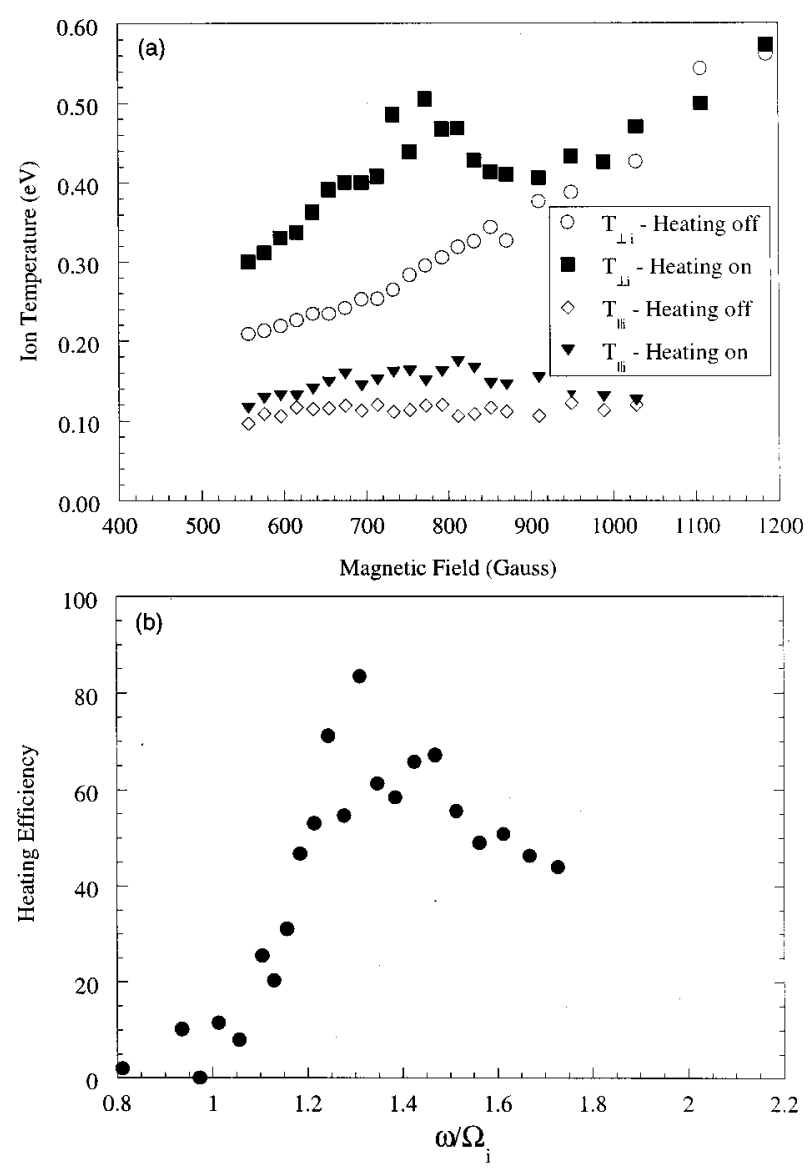

FIG. 2. At a neutral pressure of $3.0 \mathrm{mTorr}$ and heating power of $200 \mathrm{~W}$ with $19 \mathrm{~cm}$ helicon driving antenna. (a) The perpendicular and parallel ion temperatures with the heating circuit on and off vs the magnetic field strength. (b) The perpendicular ion heating efficiency vs $\omega / \Omega_{i}$.

of 12 gauge wire oriented so that the axes of the each of the two coils were transverse to the background axial field. This antenna geometry is similar to a Nagoya III (Ref. 27) configuration.

\section{ION HEATING RESULTS}

For the $19 \mathrm{~cm}$ helicon driving antenna and a neutral pressure of 3 mTorr, the on-axis, perpendicular and parallel ion temperatures as a function of magnetic field are shown in Fig. 2(a). These results are for $200 \mathrm{~W}$ of power applied to the heating antenna circuit (not the helicon driving antenna). The amount of power coupled to the plasma is not determined, although the reflected power is roughly 5\%. Figure 2(b) shows the perpendicular heating efficiency, ( $T_{i}$ heating on $\left.-T_{i \text { heating off }}\right) / T_{i \text { heating off }}$, vs the ratio of the heating rf frequency to the cyclotron frequency, $\omega / \Omega_{i}$. Figure 3 shows the same data for a neutral pressure of 1.5 mTorr. In both of these cases, the ion heating is clearly anisotropic, $\Delta T_{\perp i} / \Delta T_{\| i}>1$, and the maximum heating efficiency occurs between the first and second cyclotron harmonics. At the lower neutral pressure, 1.5 mTorr, the heating efficiency is $800 \%$, but when the neutral pressure is increased to 3.0 mTorr the heating efficiency drops to $80 \%$. The change in 

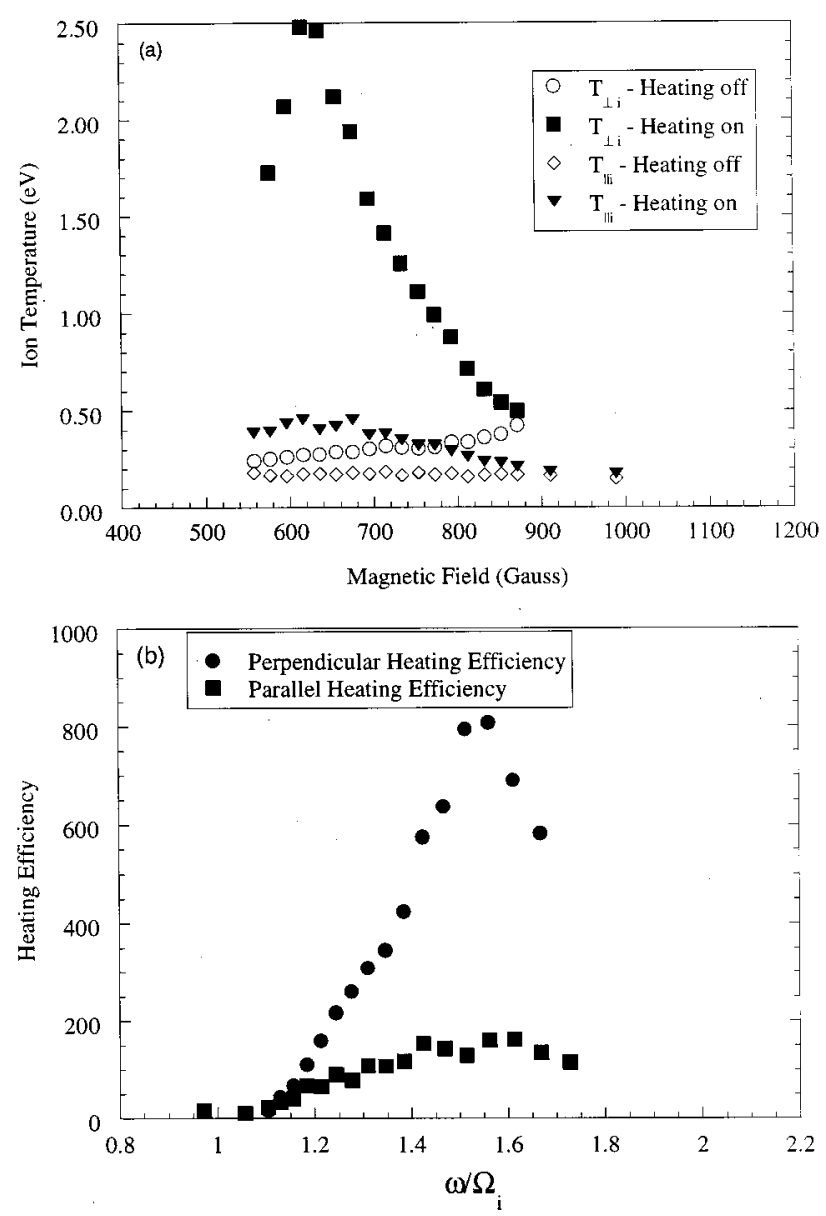

FIG. 3. At a neutral pressure of $1.5 \mathrm{mTorr}$ and heating power of $200 \mathrm{~W}$ with $19 \mathrm{~cm}$ helicon driving antenna. (a) The perpendicular and parallel ion temperatures with the heating circuit on and off versus the magnetic field strength. (b) The perpendicular and parallel ion heating efficiencies vs $\omega / \Omega_{i}$.

neutral pressure in the helicon source causes a transition from the inductive mode to the helicon mode. ${ }^{2}$ The density increases from $n \sim 5 \times 10^{12} \mathrm{~cm}^{-3}$ to $n \sim 5 \times 10^{13} \mathrm{~cm}^{-3}$ as the neutral pressure increases from 1.5 mTorr to 3.0 mTorr for the helicon source operating at the same power and magnetic field strength. The unheated ion temperatures remain the same. This change in density increases the ion-ion collision frequency by an order of magnitude.

Figures 4 and 5 show the same data as Figs. 2 and 3 for neutral pressures of 3.0 mTorr and 1.5 mTorr, respectively. In these cases, a $35 \mathrm{~cm}$ long helicon driving antenna was used. The $3.0 \mathrm{mTorr}$ data for the $35 \mathrm{~cm}$ helicon antenna looks similar to the 3.0 mTorr data with the $19 \mathrm{~cm}$ helicon driving antenna. The maximum heating efficiency lies between the first and second cyclotron harmonics and the heating efficiency is half that of the $19 \mathrm{~cm}$ antenna case. The 1.5 mTorr data for the $35 \mathrm{~cm}$ driving antenna is significantly different from the $19 \mathrm{~cm}$ antenna data. The heating efficiency is not peaked, but increases with decreasing magnetic field. Note that $T_{i}$ without ion heating does not increase linearly with the magnetic field as it did in the other three cases. The linear increase of $T_{i}$ in HELIX with magnetic field was reported earlier by Scime et al. ${ }^{8}$ Since the ion heating effi-
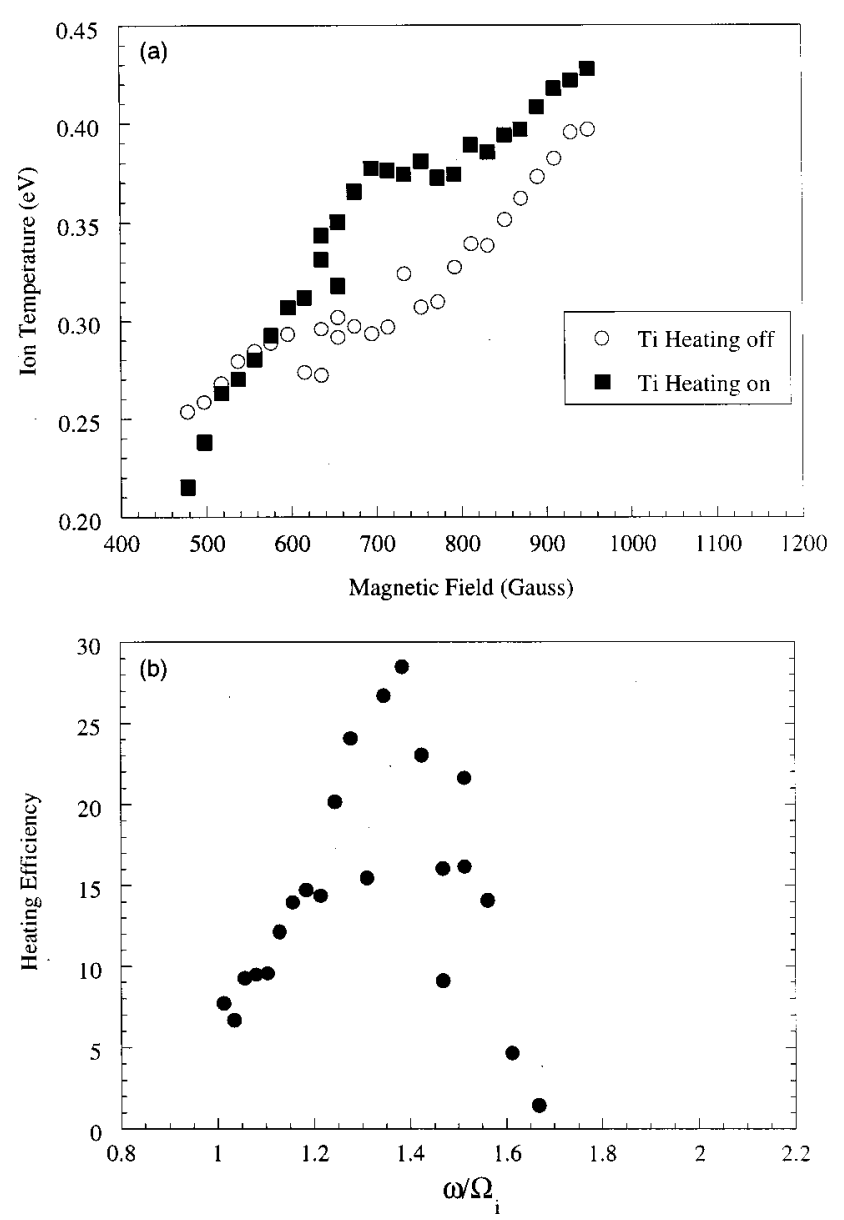

FIG. 4. At a neutral pressure of $3.0 \mathrm{mTorr}$ and heating power of $200 \mathrm{~W}$ with $35 \mathrm{~cm}$ helicon driving antenna. (a) The perpendicular ion temperatures with the heating circuit on and off versus the magnetic field strength. (b) The perpendicular ion heating efficiency vs $\omega / \Omega_{i}$.

ciency does not depend soley on the heating frequency to gyro frequency ratio, this suggests that the ion heating is not a wave particle resonant frequency effect. Instead, the ion heating may peak for the helicon source parameters that provide the best conditions for wave propagation. It is worth noting that the rapid increase in intrinsic ion temperature seen in Fig. 5 is not accompanied by visual changes in the plasma that are typically associated with helicon mode transitions. Additional wave measurements would be needed to determine if the temperature increase is due to a change in the structure of the helicon wave.

\section{WAVE PROPAGATION RESULTS}

The time dependent magnetic field of the heating antenna results in electric field components both perpendicular and parallel to the magnetic field. Based on the HELIX plasma parameters and results from other experiments using similar antenna geometries ${ }^{28}$ this antenna is likely to launch an electrostatic ion cyclotron (EIC) wave perpendicular to the magnetic field and an ion acoustic wave along the magnetic field. The short perpendicular wavelength approximation for the EIC wave dispersion relation is ${ }^{29}$ 

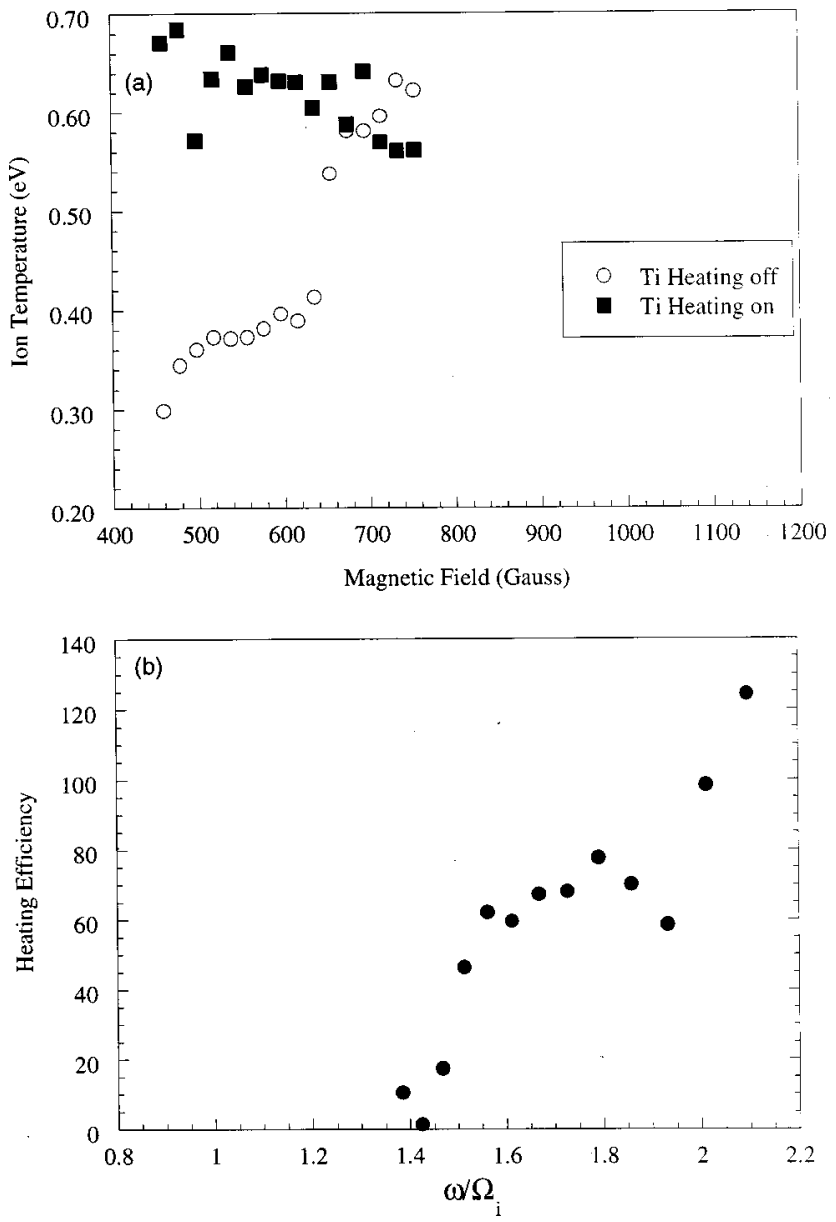

FIG. 5. At a neutral pressure of $1.5 \mathrm{mTorr}$ and heating power of $200 \mathrm{~W}$ with $35 \mathrm{~cm}$ helicon driving antenna. (a) The perpendicular ion temperatures with the heating circuit on and off versus the magnetic field strength. (b) The perpendicular and parallel ion heating efficiencies vs $\omega / \Omega_{i}$.

$$
\omega^{2}=\Omega_{i}^{2}+k^{2} C_{s}^{2}
$$

where $\Omega_{i}$ is the ion gyrofrequency, $k$ is the wave number, and $C_{s}$ is the sound speed. Note that the heating in all of the experimental cases goes to zero as the wave frequency approaches the cyclotron frequency. This is consistent with the dispersion relation as $\omega<\Omega_{i}$ yields $k^{2}<0$, i.e., no wave propagation. According to Eq. (1), the perpendicular wavelength for the peak heating at 1.5 mTorr with the $19 \mathrm{~cm}$ helicon driving antenna is $\lambda \sim 9.7 \mathrm{~cm}$ for the measured HELIX parameters. Such a wave would fit within HELIX.

It has been demonstrated that LIF measurements of the first order perturbation of the ion distribution function can be used to measure the perpendicular wavelength for EIC waves. ${ }^{21,22}$ Figure 6 shows the real and imaginary parts of the perturbed ion distribution function measured at 1.5 mTorr for the $35 \mathrm{~cm}$ helicon driving antenna and a HELIX magnetic field of $556 \mathrm{G}$. Assuming that the waves affecting the ion distribution are low frequency electrostatic waves, the first order perturbation to the ions in the case of weak collisions is given by Safarty et al., ${ }^{22}$
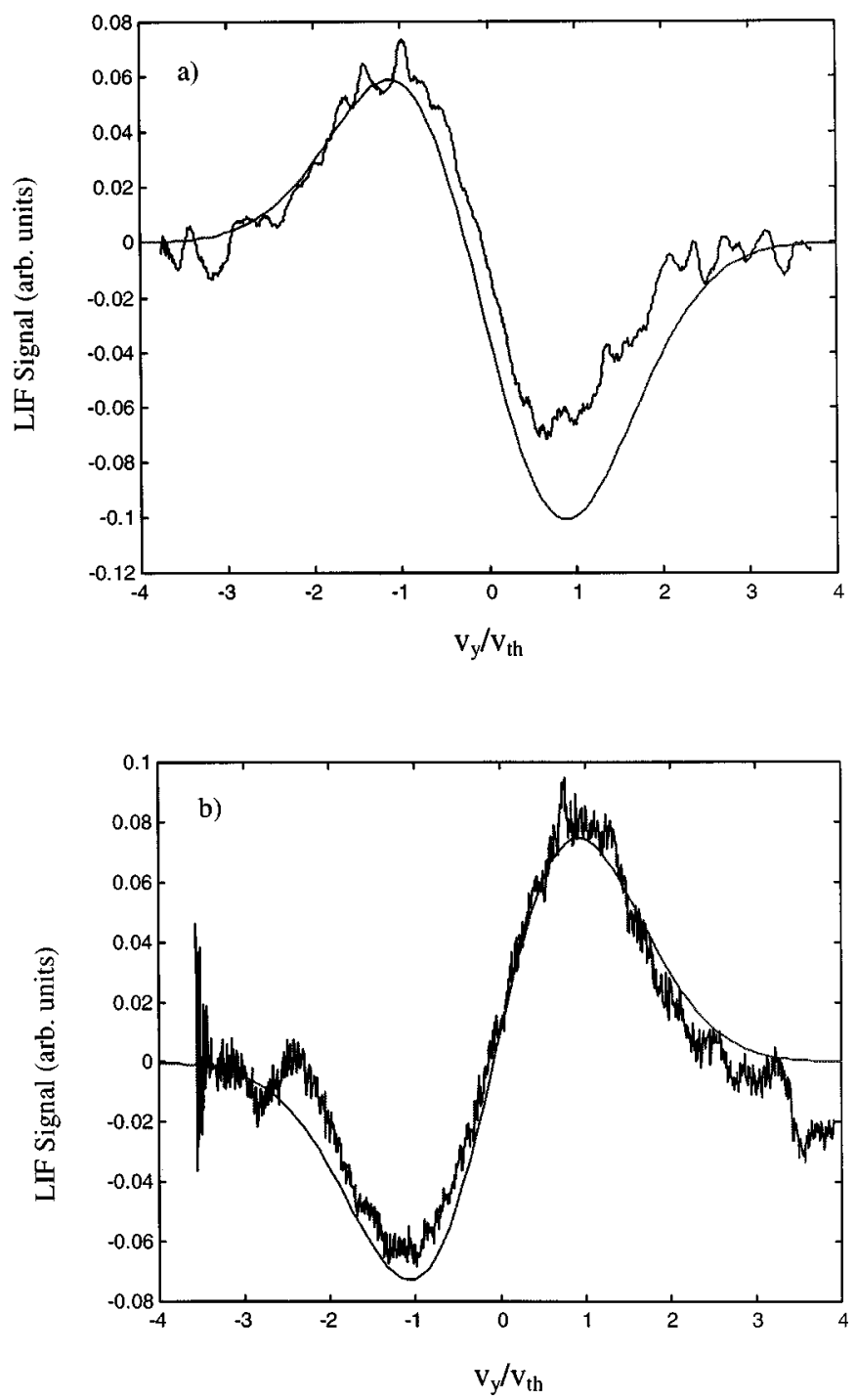

FIG. 6. For a neutral pressure of $1.5 \mathrm{mTorr}$ and $200 \mathrm{~W}$ of heating power with the $35 \mathrm{~cm}$ helicon driving antenna. (a) The measured and theoretically derived real part of the first order perturbation to the velocity distribution functions. (b) The measured and theoretically derived imaginary part of the first order perturbation to the velocity distribution function.

$$
\begin{aligned}
f_{1}\left(\mathrm{v}_{y}\right)= & \left(\frac{-e \Phi_{1}}{\pi M \nu_{t h \|}^{2}}\right) f_{0}\left(\mathrm{v}_{y}\right) \\
& \times \sum_{n, m}\left(\left(1+Z\left(\zeta_{n+m}\right)\left(\zeta_{0} \frac{T_{\|}}{T_{\perp}}+\left(1-\frac{T_{\|}}{T_{\perp}}\right) \zeta_{n-m}\right)\right)\right. \\
& \times J_{m}\left(\frac{k_{\perp} \nu_{y}}{\Omega_{i}}\right) e^{-i m \pi / 2} e^{i(m+n) \theta} e^{i k_{x} \nu_{y} / \Omega_{i}} e^{-a^{2} / 8} e^{-c^{2} / 4} \\
& \times \sum_{l} I_{(n+l) / 2}\left(\frac{a^{2}}{8}\right) I_{l}\left(\frac{a c}{2}\right) e^{i l \pi / 2}
\end{aligned}
$$

with

$$
\begin{aligned}
& a=\sqrt{2} k_{\perp} \rho, \\
& c=\sqrt{2} k_{\perp} \rho \sin \theta,
\end{aligned}
$$




$$
\begin{aligned}
& Z\left(\zeta_{n}\right)=\frac{1}{\sqrt{\pi}} \int d s \frac{e^{-s^{2}}}{s-\zeta_{n}}, \\
& \zeta_{n}=\omega_{\mathrm{eff}}-n \Omega_{i}-k_{\|} \mathrm{\vee}_{0} .
\end{aligned}
$$

Here $k_{x}$ and $k_{y}$ are the wave numbers along the Cartesian axes perpendicular to the magnetic field and $k_{\perp}^{2}=k_{x}^{2}+k_{y}^{2} . \rho$ is the average Larmor radius, $Z$ is the plasma dispersion function. $\Omega_{i}$ is the ion cyclotron frequency, $\mathrm{v}_{0}$ is the parallel drift velocity, and $\omega_{\text {eff }}$ is the effective wave frequency defined as $\omega_{\text {eff }}=\omega+i \nu$. The effective frequency includes a collision term, $i \nu$, from the Fokker-Planck model including weak collisions used by Safarty et al $^{22}$ in the $f_{1}(\mathbf{v})$ derivation. $k_{\|}$is eliminated in terms of $k_{\perp}$ using the complete solution to the electrostatic wave dispersion relationship ${ }^{22}$

$$
\begin{aligned}
k^{2} \epsilon\left(k_{\|}, k_{\perp}, \omega\right)= & k_{\|}^{2}+k_{\perp}^{2}+\sum_{i, e} k_{d s}^{2}\left(1+\sum_{n} e^{-a_{s} I_{n}\left(a_{s}\right) Z\left(\zeta_{n}\right)}\right. \\
& \left.\times\left(\zeta_{n}+\frac{n \Omega T_{\|}}{\sqrt{2} k_{\|} \nu_{t h \|} T_{\perp}}\right)\right)=0
\end{aligned}
$$

where $k_{d s}$ is the Debye wave number for species $s=e, i$, and $a_{s}=\left(k_{\perp} \rho_{s}\right)^{2} .^{22}$ This dispersion relationship contains both EIC and Bernstein wave solutions. For $k_{\perp} \rho \ll 1$, the EIC wave root to the dispersion relationship is satisfied. For $k_{\perp} \rho \sim 1$, the ion Bernstein wave root is satisfied.

The real and imaginary parts of $f_{1}\left(\mathbf{v}_{y}\right)$ can be numerically solved according to Eq. (2). ${ }^{22}$ Model curves based on Eq. (2) and the full kinetic EIC dispersion relationship are shown as smooth, thin, solid lines in Fig. 6. Values of $k_{\perp}$ $\sim 0.150 \mathrm{~cm}^{-1}$ and $k_{\|} \sim 0.404 \mathrm{~cm}^{-1}$ were used to generate the model curves. All other parameters were calculated using the measured HELIX parameters. The agreement between the measured real and imaginary parts of the first order perturbed distribution function and the theoretically predicted curves strongly support the conclusion that the heating antenna launches an EIC wave into the helicon source. The zeroth order velocity distribution functions for the perturbed velocity distribution measurements were fully thermallized Gaussian distributions that show no evidence of bulk ion motion in the wave fields of the heating antenna. This means the measurements of $f_{1}\left(\mathrm{v}_{y}\right)$ are of true wave perturbations to the velocity distribution. Given $k_{\perp} \sim 0.150 \mathrm{~cm}^{-1}, k_{\perp} \rho \ll 1$ is satisfied in the HELIX as expected for an EIC wave. For $k_{\perp}$ $\sim k_{\|}$, the phase velocity should be comparable to the ion sound speed $C_{s} \cdot{ }^{29}$ For $\omega=230 \mathrm{krad} / \mathrm{s}$ and $k_{\|}=0.404 \mathrm{~cm}^{-1}$, $\omega / k=\sqrt{k T_{e} / m_{i}}$ yields an electron temperature, $T_{e}$, of $4.5 \mathrm{eV}$, a reasonable value for HELIX.

Although fitting the real and imaginary parts of $f_{1}(\mathbf{v})$ to the model provides both the wavelength and amplitude of the waves, it is not necessary to use the model equations to determine the amplitude of the electrostatic wave. For low frequency electrostatic waves, the ratio of the perturbed density to the zeroth order density is given by

$$
\frac{n_{1}}{n_{0}} \approx \frac{e \phi_{1}}{k_{b} T_{e}},
$$

where $e$ is the electron charge, $k_{b}$ is Boltzmann's constant, $\phi_{1}$ is the electrostatic perturbation, $n_{1}$ is the perturbed density, and $n_{0}$ is the ion density. Thus, the ratio of $n_{1}$ to $n_{0}$ can be used as a measure of the electrostatic potential perturbation. Using LIF to measure $f_{0}(\mathbf{v})$ and $f_{1}(\mathbf{v})$, the zeroth and first order densities can be obtained by integrating $f_{0}(\mathbf{v})$ and $f_{1}(\mathbf{v})$ over all of velocity space,

$$
\begin{aligned}
& n_{0}=\int_{-\infty}^{\infty} d \mathbf{v}^{3} f_{0}(\mathbf{v}), \\
& n_{1}^{2}=\left(\int_{-\infty}^{\infty} d \mathbf{v}^{3} \operatorname{Re}\left\{f_{1}(\mathbf{v})\right\}\right)^{2}+\left(\int_{-\infty}^{\infty} d \mathbf{v}^{3} \operatorname{Im}\left\{f_{1}(\mathbf{v})\right\}\right)^{2} .
\end{aligned}
$$

A similar measurement, but less sensitive to statistical errors, uses the average perturbed velocity. Linearization of the ion continuity equation gives

$$
\frac{n_{i 1}}{n_{i 0}}=\frac{k}{\omega} \mathrm{v}_{i 1} .
$$

The average ion velocity can be found from the perturbed ion distribution function

$$
\left\langle\mathbf{v}_{i 1}\right\rangle^{2}=\left(\int_{-\infty}^{\infty} d \mathbf{v}^{3} \mathbf{v} \operatorname{Re}\left\{f_{1}(\mathbf{v})\right\}\right)^{2}+\left(\int_{-\infty}^{\infty} d \mathbf{v}^{3} \mathbf{v} \operatorname{Im}\left\{f_{1}(\mathbf{v})\right\}\right)^{2}
$$

and substituting into Eq. (4) yields

$$
\frac{\left\langle\mathrm{v}_{i l}\right\rangle}{C_{s}}=\frac{e \phi_{1}}{k_{b} T_{e}} .
$$

Thus, the average perturbed ion velocity can be used as a measure of the amplitude of the EIC wave in the plasma. Because the integrands become even functions of velocity, the measured average perturbed velocity is less sensitive to small errors in the first order ion velocity distribution than the perturbed density measurement. The odd integrands in Eq. (6) lead to the differencing of two large numbers to obtain a small result. At this time, the statistical noise in the measurement of $f_{1}$ are not reliable enough to make the potential measurements based on Eq. (7). Langmuir probe measurements of $n_{i 1} / n_{i 0}$, using the ion saturation current, are impractical because such probes cannot survive the high density, steady state helicon plasma without contaminating the optical windows used for LIF measurements.

Figure 7 shows the ion heating efficiency as a function of the electrostatic potential inferred from the average, onaxis, perturbed ion velocity obtained by varying the magnetic field strength in HELIX at 1.5 mTorr using the $35 \mathrm{~cm}$ helicon driving antenna (the same scan shown in Fig. 5). Given that $T_{e}$ remains roughly constant, the electrostatic potential is clearly anticorrelated with the ion heating efficiency, i.e., maximum heating occurs for the smallest wave amplitude. Since the input power from the antenna is held constant, either the wave is coupled to the plasma less efficiently or the wave energy is being absorbed more efficiently by the ions when the heating is maximum. Less efficient wave coupling seems unlikely given that the ion temperature increases by nearly an order of magnitude as the wave amplitude de- 


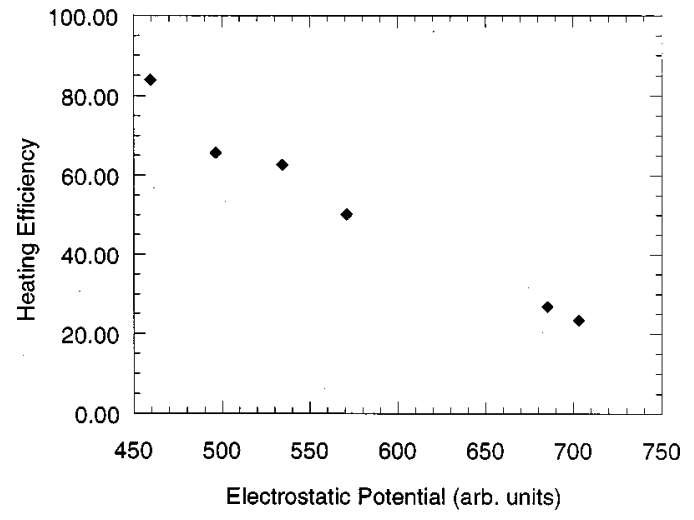

FIG. 7. For a neutral pressure of $1.5 \mathrm{mTorr}$ and $200 \mathrm{~W}$ of heating power with the $35 \mathrm{~cm}$ helicon driving antenna; the amplitude of the electrostatic potential vs heating efficiency.

creases (Fig. 7). However, the measurement of a smaller wave amplitude at a fixed position may also indicate that the location of the ion heating changes with changes in the helicon source parameters.

The current experimental apparatus is capable of radially resolved LIF measurements over $\pm 0.5 \mathrm{~cm}$ from the axis of HELIX. However, attempts to determine if the wave is being absorbed as it penetrates to the center by measuring the radial profile of $\left\langle\mathrm{v}_{1}\right\rangle$ have been equivocal. Future experiments are expected to have significantly better radial access and the $\left\langle\mathrm{v}_{1}\right\rangle$ profile experiments will be revisited at that time.

\section{CONCLUSION}

In conclusion, we have demonstrated ion heating in the HELIX source. Ion temperature increases of as much as $800 \%$ and strongly anisotropic ion heating are observed. Measurements of the perturbed ion velocity distribution function confirm the existence of an EIC wave. Since this is the only known additional free energy source, we believe that the ion heating results from the absorption of energy from the electrostatic ion cyclotron wave. The most likely explanation of the heating efficiency dependence on the helicon source magnetic field and neutral pressure is that different magnetic field strengths result in different source parameters that change the coupling to, and the propagation of, the EIC wave in the helicon source. In some cases, a very narrow, resonantlike, regime allows very efficient coupling of the ion heating system to the helicon source. This results in large increases in ion temperature over a narrow range of source magnetic fields. In other cases, an ion temperature increase is seen over a broader range of HELIX parameters. With this ion heating system, some control over the temperature anisotropy is possible over a variety of HELIX operating parameters.

\section{ACKNOWLEDGMENTS}

The authors would like to thank Tom Lovell for loan of the RF capacitors. Mike Mauel for suggesting the transverse antenna geometry, Fred Skiff for extremely helpful information concerning measurements of the first order perturbation to the velocity distribution function and EIC wave, and the WVU Q-machine group for developing the LIF apparatus at WVU. Also a special thanks to Sergio DeSouza-Machado for the $f_{1}\left(\mathbf{v}_{y}\right)$ curve fitting routines. This work supported by the U.S. Department of Energy under Grant No. DE-FG0297ER54420 and the National Science Foundation under Grant No. ATM-9616467.

${ }^{1}$ A. W. Degeling and R. W. Boswell, Phys. Plasmas 4, 2748 (1997).

${ }^{2}$ F. F. Chen, J. Vac. Sci. Technol. A 10, 1389 (1992).

${ }^{3}$ R. T. Chen and N. Hershkowitz, Phys. Rev. Lett. 80, 4677 (1998),

${ }^{4}$ M. Light, I. D. Sudit, F. F. Chen, and D. Arnush, Phys. Plasmas 2, 4094 (1995).

${ }^{5}$ J-H Kim, S-M. Yun, and H-Y. Chang, Phys. Lett. A 221, 94 (1996).

${ }^{6}$ A. R. Ellingboe and R. W. Boswell, Phys. Plasmas 3, 2797 (1996).

${ }^{7}$ M. G. Shats, D. L. Rudakov, R. W. Boswell, and G. G. Borg, Phys. Plasmas 4, 3269 (1997).

${ }^{8}$ E. Scime, P. A. Keiter, M. W. Zintl, M. M. Balkey, J. L. Kline, and M. E. Koepke, Plasma Sources Sci. Technol. 7, 186 (1998).

${ }^{9}$ P. A. Keiter, E. E. Scime, and M. M. Balkey, Phys. Plasmas 4, 2741 (1997).

${ }^{10}$ B. J. Anderson, S. A. Fuselier, S. P. Gary, and R. E. Denton, J. Geophys. Res. 99, 5877 (1994).

${ }^{11}$ S. P. Gary, Space Plasma Instabilities (Cambridge, New York, 1993).

${ }^{12}$ John Howard Australian National University (private communication).

${ }^{13}$ B. B. Kadomtsev, Tokamak Plasma: A Complex Physical System (IOP, Bristol. Philadelphia, 1992).

${ }^{14}$ D. J. Rose and M. Clark Jr., Plasmas and Controlled Fusion (Wiley, MIT Press, New York, London, 1961).

${ }^{15}$ T. N. Good, H. R. Thompson, Jr., and N Rynn, Phys. Fluids 31, 1237 (1988).

${ }^{16}$ R. A. Cairns, Radio-Frequency Heating of Plasmas (Adam Hilger, Bristol, Philadelphia, and New York, 1991).

${ }^{17}$ B. Grek and M. Porkolab, Phys. Rev. Lett. 30, 836 (1973).

${ }^{18}$ M. Ono, Phys. Fluids B 5, 241 (1993).

${ }^{19}$ R. A. Stern, D. N. Hill, and N. Rynn, Phys. Rev. Lett. 37, 833 (1981).

${ }^{20}$ R. McWilliams and D. Sheehan, Phys. Rev. Lett. 56, 2485 (1986).

${ }^{21}$ F. Skiff and F. Anderegg, Phys. Rev. Lett. 59, 896 (1987).

${ }^{22}$ M. Sarfaty, S. De Souza-Machado, and F. Skiff, Phys. Plasmas 3, 4316 (1996).

${ }^{23}$ D. H. Hill, S. Fornaca, and M. G. Wickham, Rev. Sci. Instrum. 54, 309 (1983).

${ }^{24}$ John L. Kline, M.S. thesis, West Virginia University, 1998.

${ }^{25}$ David A. Edrich, Ph.D. thesis, UC-Irvine, 1995.

${ }^{26}$ T. H. Stix and W. R. Palladino, Phys. Fluids 1, 446 (1958).

${ }^{27}$ S. Okamura, K. Adati, T. Aoki, D. R. Baker, H. Fujita, H. R. Garner, K. Hattori, S. Hidekuma, T. Kawamoto, R. Kumazawa, Y. Okubo, and T. Sato, Nucl. Fusion 26, 1491 (1986).

${ }^{28}$ F. Skiff, M. Ono, and K. L. Wong, Phys. Fluids 31, 2030 (1988).

${ }^{29}$ F. F. Chen, Plasma Physics and Controlled Fusion (Plenum, New York, 1984). 